\title{
Stellar evolution models with mass loss and turbulence
}

\author{
M. Vick ${ }^{1,2}$, G. Michaud ${ }^{1}$ and O. Richard ${ }^{2}$ \\ ${ }^{1}$ Département de Physique, Université de Montréal, Montréal, PQ, H3C 3J7 \\ email: mathieu.vick@umontreal.ca,michaudg@astro.umontreal.ca \\ ${ }^{2}$ GRAAL, Université Montpellier II, CNRS, Place E. Bataillon, 34095 Montpellier \\ Cedex, France \\ email: Olivier.Richard@graal.univ-montp2.fr
}

\begin{abstract}
Although chemical separation is generally accepted as the main physical process responsible for the anomalous surface abundances of AmFm stars, its exact behavior within the interior of these stars is still uncertain. We will explore two hydrodynamical processes which could compete with atomic diffusion: mass loss and turbulence. We will also discuss the extent to which separation occurs immediately below the surface convection zone as well as the extent to which separation occurs below 200,000 K. To do so, self-consistent stellar models with mass loss and turbulence where calculated using the Montreal stellar evolution code and compared to observations of $\mathrm{A}$ and $\mathrm{F}$ stars. It is shown that to the precision of observations available for F stars, a mass loss rate of $2 \times 10^{-14} M_{\odot} \cdot \mathrm{yr}^{-1}$ is compatible with observations and that no turbulence is then required.
\end{abstract}

Keywords. stars: evolution, stars: abundances, stars: mass loss, turbulence

\section{Introduction}

On the main sequence, most if not all slowly rotating and non magnetic A and early type $\mathrm{F}$ stars are believed to have anomalous surface abundances. These chemically peculiar stars are classified as AmFm stars (Preston 1974) and typically have observed underabundances of $\mathrm{CNO}, \mathrm{Sc}$ and $\mathrm{Ca}$ as well as overabundances of iron peak elements and rare earths (see Cayrel, Burkhart, \& Van't Veer 1991). In these stars, the surface convection zone is sufficiently shallow for the effect of a relatively slow process, such as atomic diffusion, to manifest itself at the the stellar surface. Potentially more efficient processes, such as convective, rotational or turbulent mixing as well as large scale processes such as mass loss, can slow the effect of atomic diffusion, thereby reducing surface abundance anomalies. In order to quantitatively reproduce observed surface abundances, models with atomic diffusion must consider one of these aforementioned inhibiting processes in order to reduce predicted anomalies which are larger (by a factor of 2-5) than those observed (Turcotte, Michaud, \& Richer 1998).

In Richer, Michaud, \& Turcotte (2000), diffusion models which include turbulent mixing are able to reproduce the observed surface abundance anomalies for AmFm stars of many open clusters. These same models have also successfully explained observed abundances in Pop II (Richard, Michaud et \& Richer 2005, Korn et al. 2006) and horizontal branch stars (Michaud, Richer, \& Richard 2007, Michaud, Richer, \& Richard 2008). In the AmFm models as well as the Pop II models, mixing is enforced in such a way that chemical separation occurs deeper than 200,000 K. Around this temperature, an iron convection zone naturally appears for models of at least $1.5 M_{\odot}$ (this also depends on the amount of mixing which tends to suppress it). In contrast, another explanation for 
AmFm stars suggests that chemical separation is to occur below the surface convection zone (SCZ). Both these scenarios will be discussed.

The effects of mass loss on the surface abundances of these stars have also been previously investigated by Michaud et al. (1983), Michaud \& Charland (1986), Alecian (1996) and Leblanc \& Alecian (2008). However, a further investigation is warranted since these studies analyzed static stellar models which included a limited number of elements.

In the following we will look at the effects of mass loss and turbulence both at the surface and within the stable radiative zone of these stars. We will start by detailing our models $(\S 2)$ as well as our treatment of mass loss $(\S 3)$. The following sections will compare the effects of mass loss and turbulence on the internal profiles $(\S 4)$ and on surface abundances $(\S 5)$.

\section{Stellar Evolution Models}

These 1D stellar evolution models were calculated as described in Turcotte et al. (1998b) (see also Richard, Michaud, \& Richer 2001 and references therein). Models were evolved from the homogeneous pre-main sequence up to the bottom of the sub giant branch with the abundance mix listed in Table 1 of Turcotte et al. (1998b). Radiative accelerations are calculated at each time step and at each mesh point for 28 chemical species. The atomic data is taken from the OPAL database (Iglesias \& Rogers 1996). The Rosseland mean opacity is also continuously updated which means that the treatment of chemical transport is completely self-consistent. The corrections for redistribution of momentum are from Gonzalez et al. (1995) and LeBlanc, Michaud \& Richer (2000). The atomic diffusion coefficients are taken from Paquette et al. (1986). Semi-convection is included as described in Richard et al. (2001), following Kato (1966), Langer, El Eid \& Frick (1985) and Maeder (1997). The Krishna-Swamy $T-\tau$ relation (Krishna-Swamy 1966) is used as the boundary condition in the atmosphere. The corresponding value of the mixing length parameter, which is calibrated to fit the radius and luminosity of the Sun is $\alpha=2.096$ (model $\mathrm{H}$ of Turcotte et al. 1998b) with the initial mass fraction of He set to $Y_{0}=0.27769$. Models were calculated from $1.30 M_{\odot}$ to $1.55 M_{\odot}$, with mass loss rates ranging from $10^{-14}$ to $10^{-12} M_{\odot} \cdot \mathrm{yr}^{-1}$.

\section{Treatment of Mass Loss}

The mass loss is assumed spherical and chemically homogeneous. Mass loss is introduced as described in Charbonneau (1993). The resulting transport equation is:

$$
\begin{aligned}
\rho \frac{\partial c}{\partial t}= & -\nabla \cdot\left[-\rho D \nabla \ln c+\rho\left(\mathbf{U}+\mathbf{U}_{w}\right) c\right] \\
& +\rho\left(S_{n u c}+S_{w}\right) c
\end{aligned}
$$

with a Neumann condition imposed at the surface and with $\mathbf{U}_{w}$ and $S_{w}$ defined as:

$$
\begin{gathered}
\mathbf{U}_{w}=\left\{\begin{array}{l}
v_{w} \hat{\mathbf{e}}_{\mathbf{r}} \text { under the SCZ, } \\
0 \text { in the SCZ; }
\end{array}\right. \\
S_{w}=\left\{\begin{array}{l}
0 \text { under the SCZ, } \\
\frac{\dot{M}}{M_{Z C}} \text { in the SCZ. }
\end{array}\right.
\end{gathered}
$$

Here, $c$ is the time and depth dependent composition, $\rho$ density, $D$ the total diffusion coefficient, $\mathbf{U}$ the total velocity field, $\mathbf{U}_{w}$ wind velocity, $M_{C Z}$ the mass of the SCZ, $\dot{M}$ the mass loss rate, $S_{n u c}$ a source/destruction term linked to nuclear reactions and $S_{w}$ a 

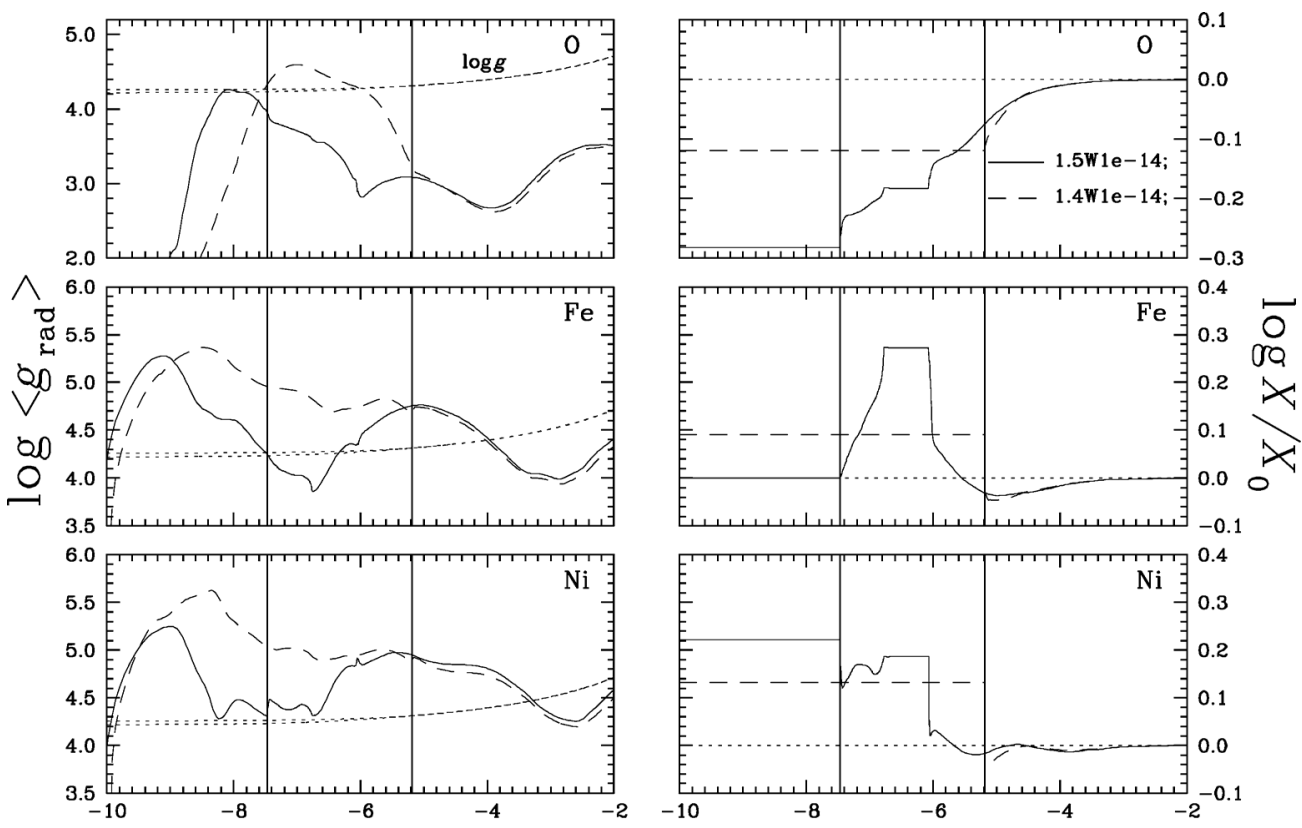

$\log \Delta M / M_{*}$

Figure 1. Left panel Radiative accelerations (continuous and dashed lines) of $\mathrm{O}, \mathrm{Fe}$ and $\mathrm{Ni}$ and gravity (dotted line) for two models of different masses at 500 Myr. Right panel Corresponding internal concentration of the three species. The models are indentified in the right panel. The number to the left $\mathrm{W}$ in the model name indicates the mass and the number to the right is the mass loss rate in $M_{\odot} \cdot \mathrm{yr}^{-1}$. The vertical lines show the position of the hydrogen-helium surface convection zone for the respective models.

destruction term linked to mass loss. The mass of the star is also continuously updated so that all quantities which depend on stellar mass are correctly calculated.

\section{Internal structure}

The anomalous surface abundances of AmFm stars are the result of chemical transport below the surface convection zone which is modulated by the competition between gravity and radiative accelerations. In Figure 1 we see how radiative accelerations and gravity vary with the mass coordinate for two models of different masses $\left(1.40 M_{\odot}, 1.50 M_{\odot}\right)$ with a mass loss rate of $10^{-14} M_{\odot} \cdot \mathrm{yr}^{-1}$. When $g$ is always greater than $g_{\mathrm{rad}}$, the element tends to sink toward the interior of the star, thus creating an underabundance at the surface, as is the case for oxygen. On the other hand, when an element is supported by the radiative field throughout most of the stellar interior (and more importantly below the surface convection zone), we can expect an overabundance of that element at the surface (as for $\mathrm{Ni}$ ). In other cases, elements accumulate where $g \sim g_{\mathrm{rad}}$. The abundance at the surface will be determined by the position of this accumulation and the bottom of the surface mixing zone (which, in the presence of turbulence, is an extension of the SCZ), since the material at its base is mixed to the surface. For the $1.50 M_{\odot}$ model, $g_{\mathrm{rad}}(\mathrm{Fe}) \operatorname{dips}$ below gravity just under the surface convection zone which causes an accumulation of iron in that region. This accumulation triples the local metal content, which leads to an increase of opacity and the appearance of an iron convection zone (which can be seen in 


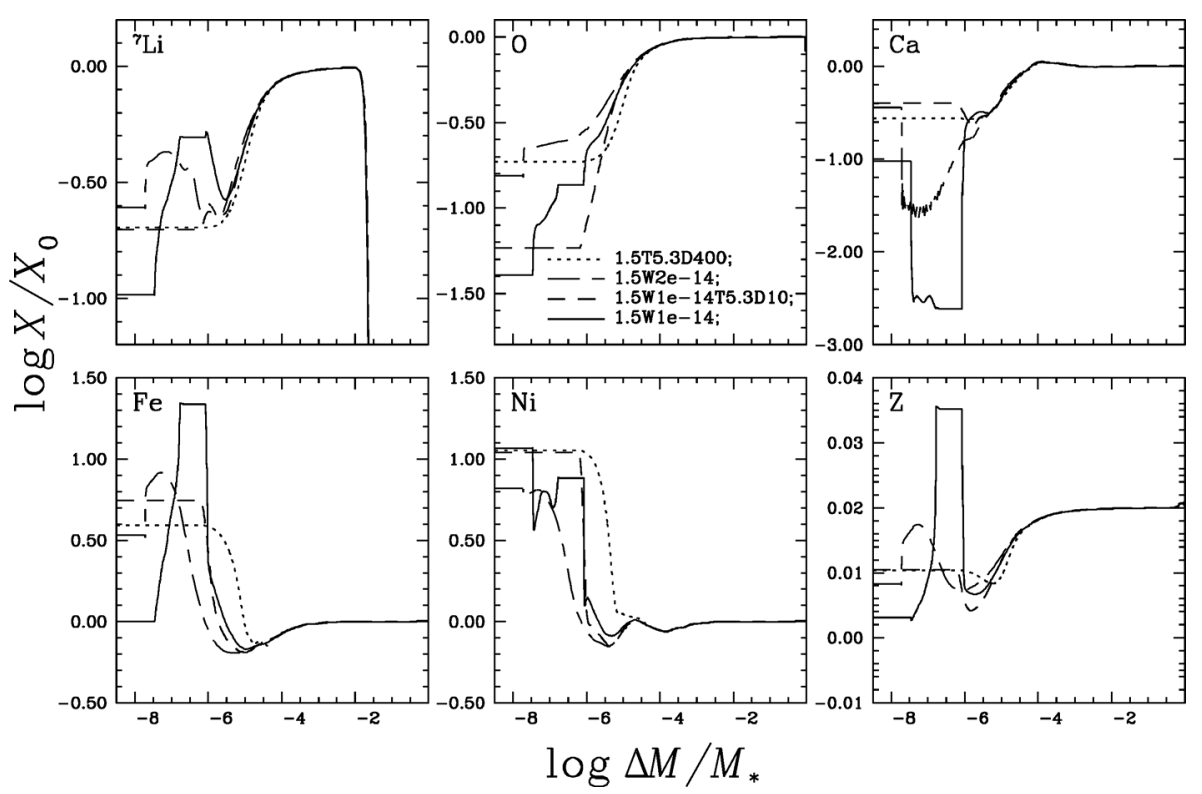

Figure 2. Abundance profiles for 4 models with turbulence and/or mass loss at $500 \mathrm{Myr}$. In the model name, the quantity to the right of $\mathrm{T}$ is the $\log$ of the temperature down to which abundances are completely homogenized by turbulence and that to the right of $\mathrm{D}$ is the coefficient multiplying the turbulent diffusion coefficient (see Richer et al. 2000). The dip in lithium around $\log \Delta M / M_{*}=-2$ is due to nuclear burning. Some numerical noise appears in the Ca profile for the $1.5 \mathrm{~W} 2 \mathrm{e} 14$ model.

the abundance profiles: the plateau between $\log \Delta M / M_{*}=6-7$ shows the homogenized abundances due to this convection zone).

In Figure 2, we see the effect of turbulence and mass loss on the abundances profiles. For all models, we see that at $500 \mathrm{Myr}$, chemical separation implies the outer $10^{-4} M_{*}$. In the models with mass loss only (1.5W1e-14 and $\left.1.5 \mathrm{~W} 2 \mathrm{e}-14\right)$, separation is allowed to occur immediately below the surface convection zone. In models with turbulence (1.5W1e-14T5.3D10 and 1.5T5.3D400), abundances are mixed down to $\log T=5.3$, which corresponds to $\log \Delta M / M_{*} \sim-6$. The only model which leads to an iron convection zone is the model with a mass loss rate of $10^{-14} M_{\odot} \cdot \mathrm{yr}^{-1}$ with no additional turbulence. This model does not generate an advective current which is strong enough to push the accumulation of iron into the surface convection zone. This also leads to a surface iron abundance which is close to its original value. However, if the wind is twice as strong $\left(2 \times 10^{-14} M_{\odot} \cdot \mathrm{yr}^{-1}\right.$, the solar value) the iron spike is weakened and pushed toward the surface. There are very significant concentration differences between the various models and this may allow to distiguish them with asterosismology.

\section{Surface Abundances}

In Figure 3, we compare surface abundances for a $1.50 M_{\odot}$ model with observed abundances of $\tau \mathrm{UMa}$ (Hui-Bon-Hoa 2000) which has an age of approximately $500 \mathrm{Myr}$ (Monier 2005 ) and $T_{\text {eff }} \sim 7000 \mathrm{~K}$ (van't Veer-Menneret \& Mégessier 1996). We tested 2 scenarios: chemical separation below 200,000 K (right panel) and the scenario in which separation occurs immediately below the surface convection zone (left panel). At this age, the surface convection zone is composed of both hydrogen and helium since helium has not yet settled gravitationally. We see that both turbulence and mass loss can effectively reduce 

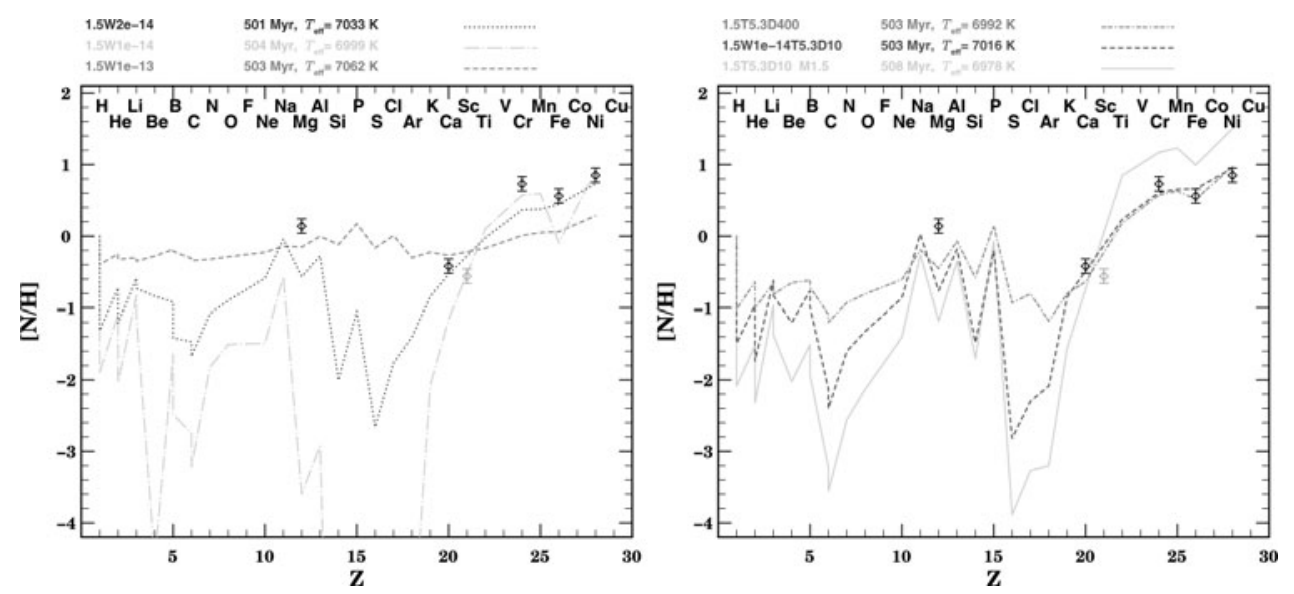

Figure 3. Observed surface abundances of $\tau$ Uma (diamonds) compared to $1.50 M_{\odot}$ models. The abundance of Sc is shown, but is not calculated in our models. Left panel Models with mass loss only; chemical separation occurs immediately below the surface convection zone. Right panel Models with turbulence; chemical separation occurs below 200,000 K.

the surface abundances to the observed levels. The model with the solar mass loss rate (1.5W2e-14) leads to a very good agreement with the data. The model with both mass loss and turbulence (1.5W1e-14D5.3D10) leads to the best fit . The mass loss rate of $10^{-13} M_{\odot} \cdot \mathrm{yr}^{-1}$ is too strong to reproduce the observations.

\section{Conclusions}

With a mass loss rate of the order of the solar mass loss rate we can successfully reproduce the observed abundance anomalies of $\tau$ UMa. We also find that with sufficiently weak winds and no turbulence, an iron convection zone naturally appears in $1.50 M_{\odot}$ models. However, for these models the surface abundance of Fe does not match the observed abundance. It is also shown that mass loss and turbulence affect abundances differently (at the surface and in the interior). It is thus possible that sufficiently precise abundance determinations could constrain the relative importance of each process. This being said, models of greater mass, where abundance determinations are more abundant, would also help in this venture. These are presently being calculated. It is still premature to favor either of the two proposed scenarios as both offer models that are able to adequately reproduce observations within the given errors.

\section{Acknowledgements}

This reasearch was partially supported at the Université de Montréal by the Natural Sciences and Engineering Researh Council of Canada. The computational resources were provided by the Réseau Québecois de Calcul de Haute Performance (RQCHP). M.Vick also thanks the Département de physique of l'Université de Montréal as well as the GRAAL of l'Université Montpellier II for financial support.

\section{References}

Alecian, G. 1996, A\&\&A, 310, 872

Cayrel, R., Burkhart, C., \& Van’t Veer, C. 1991, in IAU Symposium 145, ed. G.Michaud \& A.Tutukov

Charbonneau, P. 1993, ApJ, 405, 720 
Gonzalez, J.-F., LeBlanc, F., Artru, M.-C., \& Michaud, G. 1995, ApJ, 297, 223

Hui-Bon-Hoa, A. 2000, A\&AS, 144, 203

Iglesias, C. A. \& Rogers, F. 1996, ApJ, 464, 943

Kato, S. 1966, PASP, 18, 374

Korn, A. J., Grundahl, F., Richard, O., Barklem, P. S., Mashonkina, L., Collet, R., Piskunov, N., \& Gustafsson, B. 2006, Nature, 442, 657

Krishna-Swamy, K. S. 1966, ApJ, 145, 176

Langer, N., El Eid, M. F., \& Fricke, K. J. 1985, A\&A, 145, 179

LeBlanc, F., Michaud, G., \& Richer, J. 2000, ApJ, 538, 876

Leblanc, F., \& Alecian, G. 2008, A\& $A, 477,243$

Maeder, A. 1997, A\&A, 321, 134

Michaud, G., Tarasick, D., Charland, Y., \& Pelletier, C. 1983, ApJ, 269, 239

Michaud, G. \& Charland, Y. 1986, ApJ, 311, 326

Michaud, G., Richer, J., \& Richard, O. 2007, ApJ, 670, 1178

Michaud, G., Richer, J., \& Richard, O. 2008, ApJ, 675, 1223

Monier, R. 2005, A\&̈A, 442, 563

Paquette, C., Pelletier, C., Fontaine, G., \& Michaud, G. 1986,ApJS, 61, 177

Preston, G. W. 1974, ARAA, 12, 257

Richard, O., Michaud, G., \& Richer, J. 2001, ApJ, 558, 377

Richard, O., Michaud, G., \& Richer, J. 2005, ApJ, 619, 538

Richer, J., Michaud, G., Rogers, F., Turcotte, S., \& Iglesias, C. A. 1998, ApJ, 492, 833

Richer, J., Michaud, G., \& Turcotte, S. 2000, ApJ, 529, 338

Turcotte, S., Michaud, G., \& Richer, J. 1998a, ApJ, 504, 559

Turcotte, S., Richer, J., Michaud, G., Iglesias, C. A., \& Rogers, F. 1998b, ApJ, 504, 539

van't Veer-Menneret, C. \& Mégessier, C. 1996, A\&A A, 309, 879

\section{Discussion}

KRTIĆKA: I would suggest to calculate models of Bp stars because there you really expect both winds and chemical peculiarity.

VICK: As you say these would be very interesting to verify. Unfortunately, these hot magnetic stars outline the validity domain of our current models.

WiLlson: Is there an upper limit on $\dot{\mathrm{M}}$ from your work?

VICK: Form the models shown, which were for $15 \mathrm{M}_{\odot}$, a mass loss rate of $1 \times 10^{-13} \mathrm{M}_{\odot} / \mathrm{yr}$ completely flattens the abundances at the age of comparison (500 Myr). However, for more massive models this limiting mass loss rate increases slightly, just as it decreases for lower masses.

Christensen-DalsgandD: A naive question: what derives the wind in A and F stars?

VICK: This is actually a really good question. Cooler star winds are driven by chromospheric activity or thermal acceleration in hot coronaes (e.g., the solar wind). On the other hand, winds of hotter $\mathrm{O}$ and $\mathrm{B}$ stars are driven by radiation pressure. The importance of these processes for A and F stars isn't well known. Observational constraints are rather scarce; however, Simon et al. (1997) have detected chromospheric activity in stars of up to spectral class A7V. Whether this is the cutoff point between the 2 aforementioned processes, we still don't know.

ZAHN: Is what prevent "normal" A F stars to show abundance anomalies the presence of stars turbulence, linked with fast rotation? 
VICK: It would be fair to say that all hot $\mathrm{F}$, and A stars with sufficiently stable radiative zones will eventually show effects of atomic diffusion at their surface. The presence of strong turbulence, whether it is linked to rotation or other processes, will definitely hinder chemical separation. Mixing erases surface effects expected from atomic diffusion.

LANGER: Surface anomalies are also found in slowly rotating massive stars $\left(\rightarrow \sim 10 \mathrm{M}_{\odot}\right)$. Could those be explained by diffusion?

VICK: If the atmospheres and envelops of these stars are sufficiently stable, we should see effects of atomic diffusion. However, in these stars other processes such as strong mass loss are expected, which would impede slow processes such as microscopic diffusion. 